\title{
Correction to: Persuasive Contrastive Explanations for Bayesian Networks
}

\author{
Tara Koopman and Silja Renooij
}

\section{Correction to: \\ Chapter "Persuasive Contrastive Explanations for Bayesian Networks" in: J. Vejnarová and N. Wilson (Eds.): Symbolic and Quantitative Approaches to Reasoning with Uncertainty, LNAI 12897, https://doi.org/10.1007/978-3-030-86772-0_17}

In the originally published article, the pseudocode in Algorithm 1 does not include the lines that were left intentionally blank. This has been corrected to include 2 lines (line 9 and 14), which are intentionally left blank in the algorithm for the addition of new code to these lines. 